\title{
Three-dimensional cell culture of human mesenchymal stem cells in nanofibrillar cellulose hydrogels
}

Article

Accepted Version

Azoidis, I., Metcalfe, J., Reynolds, J., Keeton, S., Hakki, S. S., Sheard, J. and Widera, D. (2017) Three-dimensional cell culture of human mesenchymal stem cells in nanofibrillar cellulose hydrogels. MRS Communications, 7 (3). pp. 458-465. ISSN 2159-6859 doi: https://doi.org/10.1557/mrc.2017.59 Available at https://centaur.reading.ac.uk/71458/

It is advisable to refer to the publisher's version if you intend to cite from the work. See Guidance on citing.

To link to this article DOI: http://dx.doi.org/10.1557/mrc.2017.59

Publisher: Cambridge University Press

All outputs in CentAUR are protected by Intellectual Property Rights law, including copyright law. Copyright and IPR is retained by the creators or other copyright holders. Terms and conditions for use of this material are defined in the End User Agreement.

www.reading.ac.uk/centaur 
Central Archive at the University of Reading

Reading's research outputs online 
mRs Communications

\section{D Cell Culture of Human Mesenchymal Stem Cells in Nanofibrillar Cellulose Hydrogels}

\begin{tabular}{|r|l|}
\hline Journal: & MRS Communications \\
\hline Manuscript ID & MRSCOM-2017-0071.R1 \\
\hline Manuscript Type: & Special Issue Research Letter: Biomaterials for 3D Cell Biology \\
\hline Complete List of Authors: & $\begin{array}{l}\text { Azoidis, Ioannis; University of Reading, School of Pharmacy } \\
\text { Joel Metcalfe <joel.metcalfe@student.reading.ac.uk>, Joel; University of } \\
\text { Reading, School of Pharmacy } \\
\text { Reynolds, James; Lein Applied Diagnostics, Research and Development } \\
\text { Keeton, Shirley; University of Reading, School of Biological Sciences } \\
\text { Hakki, Sema; Selcuk University, Department of Periodontology } \\
\text { Sheard, Jonathan; University of Reading, School of Pharmacy; Sheard } \\
\text { BioTech Limited, R\&D } \\
\text { Widera, Darius; University of Reading, School of Pharmacy }\end{array}$ \\
\hline Keyword: & $\begin{array}{l}\text { biological, biomaterial, scanning electron microscopy (SEM), self-assembly, } \\
\text { tissue }\end{array}$ \\
\hline &
\end{tabular}




\section{D Cell Culture of Human Mesenchymal Stem Cells in Nanofibrillar Cellulose}

\section{Hydrogels}

Ioannis Azoidis ${ }^{1}$, Joel Metcalfe ${ }^{1}$, James Reynolds ${ }^{2}$, Shirley Keeton ${ }^{3}$, Sema Hakki $^{4}$, Jonathan Sheard ${ }^{1,5}$ and Darius Widera ${ }^{1, *}$

${ }^{1}$ Stem Cell Biology and Regenerative Medicine Group, School of Pharmacy, University of Reading, Whiteknights campus, Reading, RG6 6AP, United Kingdom

${ }^{2}$ Lein Applied Diagnostics Ltd, Reading Enterprise Centre, Whiteknights Rd, Reading, RG6 6BU, United Kingdom

${ }^{3}$ Cell Migration Lab, School of Biological Sciences, University of Reading, Whiteknights campus, Reading, RG6 6AP, United Kingdom

${ }^{4}$ Selcuk University Faculty of Dentistry Department of Periodontology Campus, 42079 Konya, Turkey

${ }^{5}$ Sheard BioTech Limited, Suite LP36133, 20-22, Wenlock Road London N17GU, United Kingdom

*Correspondence: Darius Widera, Stem Cell Biology and Regenerative Medicine, Reading School of Pharmacy, University of Reading, PO Box 226, Whiteknights, Reading, RG6 6AP, United Kingdom, Tel: +44(0) 1183787053

E-mail: d.widera@reading.ac.uk 


\begin{abstract}
Human mesenchymal stem cells (MSCs) are the most intensely studied and clinically used adult stem cell type. Conventional long-term cultivation of MSCs as a monolayer is known to result in a reduction of their functionality and viability. In addition, large volumes of cell culture medium are required to obtain cell quantities needed for their clinical use. In this proof of concept study, we cultivated human MSCs within a 3D nanofibrillar cellulose (NFC) hydrogel. We show that NFC is biocompatible with human MSCs, and represents a feasible approach to upscaling of their culture.
\end{abstract}




\section{Introduction}

The presence of MSCs in multiple tissue types as well as their multi-lineage differentiation and regenerative capacity has led to an increase in their clinical application with over 724 trials registered on ClinicalTrials.gov as of May 2017. The therapeutic benefit of MSCadministration in different pathologies (reviewed in [1]) can be at least partly explained by paracrine effects on endogenous regeneration that are mainly mediated by extracellular vesicles (EVs) released by MSCs [2-4]. Thus, EVs are considered to represent a costefficient alternative to transplantation of MSCs with a potentially equally beneficial therapeutic outcome.

Notably, both transplantation of MSCs and manufacturing of MSC-derived EVs require large cell numbers, long cultivation time, and consequently large volumes of cell culture medium. Thus, culture expansion in vitro is a necessary step in order to obtain sufficient quantities of cells for the intended therapeutic application. However, it is well known that during initial and extended in vitro culture expansion, MSCs accumulate chromosomal aberrations, which may be caused by the extraction of the cells from their three-dimensional endogenous niche and 2D in vitro culture conditions [5, 6]. Moreover, a large body of evidence suggests that prolonged 2D cultivation leads to a loss of multipotency and induces cellular senescence in MSCs [7]. Therefore, conventional 2D culture represents a bottleneck in a broader use of MSC-based therapeutic and the upscaling of MSC-culture is a current technological and economic challenge. .

To overcome these limitations, different 3D cultivation methods, more closely resembling the endogenous niche, have been applied to MSCs. Among others, MSCs can be cultivated in 3D within alginate hydrogels [8], collagen-based matrices [9], fibrin-poly(ester-urethane) scaffolds [10], bacteria-derived cellulose [11], methylcellulose [12] and the chondrosarcomaderived Matrigel $^{\mathrm{TM}}$ [13]. However, despite the obvious advantages over the $2 \mathrm{D}$ cultivation 
systems, most MSC 3D cultivation methods also have drawbacks. Specifically, alginate hydrogels require cross-linking, while retrieval of the cells from fibrin and collagen based matrices can only be achieved by using enzymes also affecting mammalian cells. Matrigel ${ }^{\mathrm{TM}}$ is known to be affected by batch to batch variability, cross species immunogenicity and consequently difficulties with the use in a clinical setting [14]. In contrast, similar to other bacteria-derived biologics, bacteria-derived cellulose requires cost intensive purification to avoid contaminations with endotoxins.

In this study, we examined the biocompatibility of multipotent human adipose tissue-derived and bone marrow derived mesenchymal stem cells (adMSCs and bmMSCs) with the commercially available nanofibrillar cellulose (NFC) hydrogel GrowDex®, which has been demonstrated to be biocompatible with other cell types including liver cells, liver progenitor cells, and human induced pluripotent stem cells [15-17]. We demonstrate that both adMSCs and bmMSCs interact with the NFC, are evenly distributed within all three dimensions of the hydrogel, and are viable in $0.2 \%$ and $0.5 \%$ NFC hydrogel. Moreover, MSCs were successfully differentiated in osteogenic cells within the 3D hydrogel. Finally, we show that MSCs can be easily retrieved from the 3D hydrogel via an enzymatic digestion of the NFC and that the post-NFC MSCs show similar viability to 2D pre-cultivated MSCs.

\section{Material and Methods}

\section{Nanofibrillar Cellulose and Calcofluor white staining}

NFC hydrogel (Growdex®) and cellulase solution (GrowDase ${ }^{\mathrm{TM}}$ ) were kindly provide by UPM Biochemicals, Helsinki, Finland). $0.2 \%$ NFC solution was prepared by diluting the $1.5 \%$ stock solution in DMEM (Sigma-Aldrich, Irvine, United Kingdom) and $100 \mu \mathrm{L}$ of the hydrogel was transferred into a well of a low-adhesion 96 well plate using low-retention pipette tips (Sarstedt, Leicester, United Kingdom) followed by incubation with $25 \mu \mathrm{L}$ of the Calcofluor white $(0.01 \%$ aqueous solution of Calcofluor White M2R, $1 \mathrm{~g} / \mathrm{L}$, Evans blue, $0.5 \mathrm{~g} / \mathrm{L}$, Sigma- 
Aldrich) for $20 \mathrm{sec}$ at RT. The staining was visualized using an inverted fluorescence microscope (VertA1, Carl Zeiss, Cambridge, United Kingdom) equipped with a DAPI filter.

\section{Scanning Electron Microscopy}

Cells within $0.5 \%$ NFC hydrogel or $0.5 \%$ NFC hydrogel alone were transferred into a $24 \mathrm{~mm}$

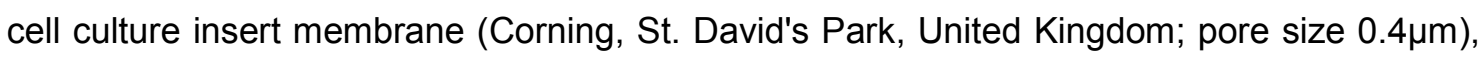
incubated for $24 \mathrm{~h}$ at $37^{\circ} \mathrm{C}$ and fixed with $2 \%$ paraformaldehyde and $2.5 \%$ glutaraldehyde for 1 hour, washed 3 times with PBS for 30 mins followed by dehydration in ethanol series then moved with a spatula on top of a stub. The samples were plunged into liquid nitrogen slash then freeze dried for 1.5 hours followed by gold coating (thickness of $25 \mathrm{~nm}$ ). Analysis was performed using a scanning electron microscope (FEI Quanta FEG 600 SEM, Thermo Fisher, Paisley, United Kingdom). Fiber size was calculated from 7 SEM images and a histogram was created. The calculation was performed using the ImageJ Fiji software. The histogram was created with GraphPad Prism software (GraphPad, La Jolla, CA, USA).

\section{Human MSCs}

Fully characterised human adipose tissue MSCs from non-diabetic adult lipoaspirates were obtained from Lonza (Slough, United Kingdom). Palatal adipose tissue MSCs and bone marrow MSCs were obtained from adult donors with written informed consent, and the study was approved by Ethics Committee of Dental Faculty of Selcuk University (approval number 2012-08). All MSCs have been characterized immunocytochemically and by tri-lineage differentiation assay as recommended by The International Society for Cellular Therapy [18].

\section{Cultivation of MSCs as 2D monolayer}

MSCs were cultivated in DMEM high glucose, 1\% L-Glutamine, 1\% penicillin/streptomycin (all from Sigma-Aldrich) and 20\% heat inactivated FBS (Sigma Aldrich, lot: 8204188981) [standard cultivation medium] at $37^{\circ} \mathrm{C}$ and $10 \% \mathrm{CO}_{2}$. Medium was changed every 2-3 days. For expansion, cells were detached by treatment with $0.05 \%$ trypsin/EDTA (Sigma-Aldrich) 
and seeded in $175 \mathrm{~cm}^{2}$ tissue culture flasks (Sarstedt). All cells were used at passages 4-13 (except palatal adMSCs for live/dead for and osteogenic differentiation assay at passage 18).

\section{Cultivation of MSCs in NFC 3D matrix}

After detachment by treatment with $0.05 \%$ trypsin/EDTA (Sigma-Aldrich), MSC suspensions were mixed with $1.5 \%$ NFC hydrogel to achieve $0.2 \%$ or $0.5 \%(\mathrm{w} / \mathrm{v})$ hydrogels with desired cell density. After 30 min of incubation at $37^{\circ} \mathrm{C}$, standard culture medium was added to the top of the hydrogel.

MSCs were cultured in $0.2 \%$ and $0.5 \%$ NFC hydrogel at the seeding density of $10^{5}$ cells/well in standard cultivation medium for up to 7 days at $37^{\circ} \mathrm{C}$ and $10 \% \mathrm{CO}_{2}$ unless otherwise stated. Medium was changed daily. Retrieval of cells from the NFC was performed by enzymatic digestion of the matrix with cellulase (GrowDase, UPM Biochemicals) for $24 \mathrm{~h}$ at $37^{\circ} \mathrm{C}$. Bright field microscopy was done using an inverted microscope (VertA1, Carl Zeiss).

\section{Liberation of MSCs from the NFC hydrogel}

MSCs were seeded at $25 \times 10^{3}$ cells per $100 \mu$ l and cultured for $96 \mathrm{hrs}$ in $0.2 \%$ and $0.5 \%$ NFC. The cellulase solution (GrowDase $\AA$, UPM, Finland) was prepared according to manufacturer's instructions. Briefly, $300 \mu \mathrm{g}$ of cellulase per $1 \mathrm{mg}$ of NFC was incubated for 4hrs at $37^{\circ} \mathrm{C}$ and $10 \% \mathrm{CO}_{2}$. Following NFC digestion, the suspension was removed and the well washed with PBS. Following centrifugation at $300 \mathrm{~g}$ for $10 \mathrm{mins}$, cell counting was performed using a haemocytometer.

\section{Cell Viability Assay (XTT)}

A cell viability assay in $2 \mathrm{D}$ and $3 \mathrm{D}$ cultures was performed using the Cell Proliferation Kit II (Sigma-Aldrich) according to the manufacturer`s instructions. BM-MSCs and palatal adMSCs were used at passage 9, and adMSCs at passage 13. The fluorescence of the XTT metabolite was measured at an excitation wavelength of $450 \mathrm{~nm}$ and a reference 
wavelength of $650 \mathrm{~nm}$ on a Spectra Max 340PC plate reader (Molecular Devices, Wokingham, United Kingdom). Measurements were taken after 7 hours of incubation.

\section{PKH67 staining and fScan 3D fluorescence imaging}

To visualize living cells within the NFC matrix, PKH67 Green Fluorescent Cell Linker Kit for General Cell Membrane Labelling (Sigma-Aldrich) was used. Briefly, $2 \times 10^{5}$ adMSCs at passage 11 were labelled according to the manufacturer's instructions and embedded within $0.5 \%$ NFC hydrogel. For detection of living, labelled cells, the fScan 488 (Lein Applied Diagnostics, Reading, UK) 3D scanning confocal microscope was used. 3D reconstruction was done using ImageJ Fiji software [19] and the Volume Viewer plugin.

\section{Live and Dead Staining and Confocal laser scanning microscopy}

AdMSCs (passage 18) cultured in $0.2 \%$ NFC on translucent TC 24 well inserts (Sarstedt), stained with Live/Dead viability/cytotoxicity kit according to the manufacturer's instruction (Thermo Fisher), fixed in 4\% PFA for 20mins and counter-stained with DAPI (Sigma-Aldrich). The TC membrane was removed from the TC insert and mounted onto a slide in Mowiol 488 mounting solution (Sigma-Aldrich). Confocal images were gathered using the Nikon A1-R inverted confocal microscope with the Nikon Plan Apo VC 20x DIC N2 optic lens, running NIS Elements AR. NFC was visualized by phase contrast (TD), DAPI at an excitation/emission of 405/450nm, Calcein 494/517nm, and Ethidium homodimer-1 at 528/617nm with the Chroma 405/488/561/647 quad mirror. A z-stack depth of $50 \mu m$ (zplane) was created for an area of $554 \times 550 \mu \mathrm{m}$ and a $3 \mathrm{D}$ reconstruction was generated with all the channels using the NIS Elements AR software (v4.0). The phase contrast channel showing the NFC was cut through the $\mathrm{Y}$ plane.

\section{Osteogenic differentiation of MSCs in 3D NFC Hydrogels}

For induction of osteogenesis, $2 \times 10^{5}$ palatal-tissue (passage 18) and liposuction-derived adMSCs (passage 14) in normal cultivation medium were embedded in $0.2 \%$ NFC and transferred to 12 well cell culture inserts (Sarstedt). Medium was changed after 3 days to 
Stem Pro Osteocyte/Chondrocyte basal medium (Life Technologies) supplemented with StemPro Osteogenesis supplement (Life Technologies) according to the suppliers instructions. In control MSCs, medium was changed to fresh standard medium. Cultures were maintained at $37^{\circ} \mathrm{C}$ and were stained with Alizarin Red $\mathrm{S}$ after 21 days of differentiation. Medium was removed and cells were washed with PBS. Fixation in $4 \%$ PFA for $30 \mathrm{~min}$ was followed by three washing steps in distilled water. Alizarin Red staining solution ( $2 \mathrm{~g}$ Alizarin Red $\mathrm{S}$ in $100 \mathrm{ml}$ distilled water, $\mathrm{pH} 4.1$ with $0.1 \% \mathrm{NH}_{4} \mathrm{OH}$, filtered) was added and incubated for $45 \mathrm{~min}$ at room temperature in the dark. The staining solution was removed and non-specific staining was removed by three washing steps with water.

\section{Statistical Analysis}

All statistical analyses were performed using GraphPad Prism software (GraphPad, La Jolla, CA, USA). Data were compared using either a student's $t$-test (two-tailed, confidence interval 95\%), or one-way analysis of variance (ANOVA) with Bonferroni correction ( $\mathrm{Cl} 95 \%$ ), where appropriate. At least 3 independent measurements were performed. $p<0.05$ was considered statistically significant.

\section{Results}

\section{NFC forms dense mesh-like structures with large pores}

In order to visualise the cellulose fibres within the 3D NFC hydrogel, an aqueous solution of Calcofluor staining was used. Subsequent fluorescence microscopy analysis clearly showed that cellulose fibres are evenly distributed within the hydrogel (Fig. 1A). To gather substructural data on the 3D NFC, the hydrogel was processed for scanning electron microscopy revealing a dense meshwork-like structure (Fig.1B). Higher magnification image showed a dense network of cellulose fibres of different size (Fig. 1C). Analysis of the fiber diameter revealed an average diameter of $2.07 \mu \mathrm{m}$ and heterogeneous distribution ranging from 0.08 to $9.1 \mu \mathrm{m}$. Notably, the majority of the fibers had a diameter smaller than $3.0 \mu \mathrm{m}$ (Fig. 1D). 


\section{D NFC hydrogels are biocompatible with MSCs}

We then explored the feasibility of cultivation of human adMSCs in three dimensional NFC hydrogels at $0.2 \%$ and $0.5 \%$ NFC hydrogels. Most MSCs in the $0.2 \%$ NFC displayed a typical round morphology (Fig. 2A). Cultivation of MSCs in $0.5 \%$ NFC resulted in a homogenous cell population with round cells similar to MSCs cultivated in $0.2 \%$ NFC. Scanning electron microscopy revealed that MSCs interact and bind to the NFC as evidenced by membrane protrusions at the matrix (Fig. 2C). In order to visualize larger numbers of living MSCs within the NFC, MSCs were labelled with PKH67 (Fig. 2D) and imaged by high content laser scanning microscopy (Fig 2E). Here, we demonstrate that living MSCs are isotropically distributed within the matrix over large areas of the well.

\section{D NFC supports high viability and proliferation of MSCs}

To assess the influence of 3D cultivation within the NFC hydrogel on cellular viability of MSCs, cells were cultured in 3D, stained with Calcein (living cells), Ethidium homodimer-1 (dead cells), fixed and counter-stained for DAPI. Subsequent laser scanning microscopy and post-hoc image analysis revealed that most cells within the hydrogel stained positively for Calcein (Fig. 3D-E). Moreover, the cells were distributed evenly in all three dimensions of the hydrogel (Fig. 3D). No pyknotic or fragmented nuclei were observed. To quantitatively determine the viability of 3D-cultivated MSCs, an XTT assay was performed (Fig. 3E). BmMSCs, liposuction adMSCs and palatal adMSCs cultivated in $0.2 \%$ NFC showed no significant changes in cellular viability compared to the 2D control. In contrast, all MSCs showed reduced viability in $0.5 \%$ NFC. However, the difference to the $2 \mathrm{D}$ control was only significant in the bmMSCs group. In order to investigate the ability of MSCs to proliferate within the NFC hydrogel $36 \times 10^{3}$ adMSCs were embedded in $0.2 \%$ or $0.5 \%$ NFC followed by cultivation for $36 \mathrm{~h}$ and XTT. A standard curve with defined cell numbers (10, 1000, 10000 , and 100000 cells) was used to normalize the XTT absorption to cell numbers. Analysis of the normalized XTT absorption values revealed that there is no significant 
difference between proliferation of MSCs as $2 \mathrm{D}$ culture and within $0.2 \%$ NFC. Note that MSCs in $0.5 \%$ proliferate although at a lower rate than in $2 \mathrm{D}$ or within $0.2 \% \mathrm{NFC}$.

\section{Viable MSCs can be retrieved from the 3D NFC hydrogel}

In order to determine if viable adMSCs can be retrieved from the 3D NFC hydrogel, cellulose was enzymatically digested using cellulase followed by harvesting of the suspension cells by centrifugation, reseeding under standard 2D conditions and a subsequent XTT assay (Fig. 3D). Here, we were able to show that adMSCs pre-cultivated in 3D show no significant differences in cellular viability to control cells (continuously cultivated as 2D monolayer).

\section{D NFC hydrogels are suitable for osteogenic differentiation of MSCs in 3D}

To assess the feasibility of osteogenic differentiation in 3D NFC, liposuction-derived adMSCs and palatal tissue-derived MSCs were subjected to differentiation in $0.2 \%$ NFC for 21 days and stained for calcium deposition using Alizarin Red S. Here, we demonstrate that human MSCs of both cellular origins deposit high amounts of calcium after 21 days of differentiation compared to undifferentiated cells in $0.2 \%$ NFC (Fig 4A-B). Note that differentiated samples show a typical deep red/magenta staining whereas undifferentiated samples and control scaffolds appear light/dim red due to unbound dye remaining in the NFC hydrogel (Fig. 4C).

\section{Discussion}

This proof-of-concept study describes for the first time 3D cultivation and differentiation of human MSCs within plant-derived 3D nanofibrillar cellulose (NFC) hydrogels. We showed that the 3D NFC features fibers and pores of different sizes and is biocompatible with human MSCs of different cellular origin. In particular, we demonstrated that living MSCs are homogenously distributed in the hydrogel and that cultivation in $0.2 \% \mathrm{NFC}$ does not negatively influence their cellular viability. Finally we demonstrated that NFC is compatible with 3D differentiation of MSCs into the osteogenic fate. 
Although MSCs represent one of the most promising adult stem cell types in regenerative medicine, their conventional expansion to obtain clinically significant cell numbers represents a significant roadblock toward routine use in the clinics. In particular, prolonged 2D cultivation of various adult stem cells harbors the intrinsic risk of chromosomal aberrations and tumorigenic transformation $[5,6,20]$. This could be at least partly explained by the removal of the stem cells from their endogenous niche which is indeed three-dimensional. In general accordance with this assumption, human breast epithelial cells were shown to develop into tumor like cells when cultured in 2D, whilst reverting to normal behavior when cultured in a more native 3D microenvironment [21]. Thus, 3D cultivation of MSCs could reduce the risk of tumorigenic transformation during expansion in vitro. In addition to the increased risk of cellular transformation, cultivation of MSCs as a $2 \mathrm{D}$ monolayer is associated with significant reduction of multipotency and acquisition of early cellular senescence. In this context, 3D cultivation of adMSCs has been shown to induce autophagy and reduce general signs of senescence [22]. Thus, 3D cultivation could provide a stable micro-environment for isolated MSCs ex vivo, maintaining their multipotency, preventing cellular senescence, and enabling high proliferation capacity and yield, whilst minimizing the culture time in vitro. In addition, cultivation of MSCs as 3D allows significantly higher cell numbers per volume of cell culture medium compared to conventional cell culture and stacks of flask-units commonly applied for large-scale production of MSCs. This would allow significant cost reduction in production of both clinically relevant numbers of MSCs and MSC-derived EVs for clinical use.

Currently, there are many commercially available 3D matrices developed to provide mechanical support and biochemical cues to enhance proliferation and differentiation of MSCs. Some matrices are fibrin or collagen based, whilst others are a varied mix of ECM molecules. For example, the mouse chondrosarcoma cell line, EHS, was used to develop the well-known and broadly utilized ECM matrix - Matrigel ${ }^{\mathrm{TM}}$ [23]. Nonetheless, Matrigel ${ }^{\mathrm{TM}}$ has some major drawbacks, such as batch to batch variability, cross species immunogenicity 
and consequently potential problems with translation into the clinic Thus, fibrin- and collagen-based 3D matrices are a well-defined alternative to Matrigel ${ }^{\mathrm{TM}}$-based $3 \mathrm{D}$ culture of MSCs. However, these natural substrates and their functionalized versions do not allow for retrieval of cells free of enzymes affecting mammalian cells. Alginates represent another 3D matrix suitable for MSC cultivation [8]. However, alginates require cross-linking prior to use.

In addition to natural materials, 3D matrices can be engineered from synthetic materials such as poly-(-I-lactic acid) nanofibres [24]. Although they offer the clear advantage of welldefined and guided construction, they need to be electrospun prior to their use which can be highly complicated and time consuming.

The plant derived native nanofibrillar cellulose (NFC) hydrogel, GrowDex®, provides a wellcharacterized and defined matrix $[17,25]$. This hydrogel is easy to handle and is biocompatible with human cells, whilst also allowing the simple retrieval of cells from the scaffold for further use [25, 26]. Although the immunogenicity of plant-derived NFC is still under investigation, the low immune response to other plant derived hydrogels has been reported $[27][12,28]$.

In contrast to the materials introduced above, the plant-derived NFC matrix harbors several crucial advantages. Firstly, as a plant derived matrix, NFC hydrogels are free of animalderived components which could facilitate their transition into clinical use. Moreover, it allows an easy retrieval of viable cells based on enzymatic digestion of the cellulose fibers using cellulases which do not affect human cells. Moreover, in contrast to other hydrogels no crosslinking is needed for the assembly of the matrix.

Our proof-of-concept study clearly shows that the commercially available and standardized 3D NFC hydrogel GrowDex ${ }^{\circledR}$ is biocompatible and supports growth of MSCs. Notably, in addition to MSCs, 3D NFC has been demonstrated to be biocompatible with human pluripotent stem cells [15] and liver cells [25]. Noteworthy, the slight decline of cellular viability of bmMSCs observed in $0.5 \% \mathrm{NFC}$ in our study, suggests that the optimal 
concentration of the NFC needs to be experimentally determined for each individual cell type (as suggested by the manufacturer). Likewise, we noted that the high concentration of 3D NFC prevented spheroid formation as similarly seen with human pluripotent stem cells [15].

In summary, this study clearly suggests that 3D NFC could be used for the development of safe and simple protocols, towards the expansion and differentiation of MSCs for routine cell culture and towards potential clinical application. In additional, 3D NFC could be used for obtaining high cell numbers required for isolation of MSC-derived extracellular vesicles.

\section{Acknowledgments}

DW is supported by a grant of the DFG (German Research Foundation, WI4318/2-1). JS is supported by UPM Biochemicals, Helsinki, Finland. SSH is supported by The Scientific and Technological Research Council of Turkey (TUBITAK/SBAG-112S587), Ankara, Turkey and Selcuk University Research Coordination Office (BAP), Konya, Turkey. We thank Prof Erdal Karaoz, Liv Hospital, Center for Regenerative Medicine and Stem Cell Research \& Manufacturing (LivMedCell), Istanbul, Turkey for providing human bone marrow MSCs. The sponsors did not participate in study design and data analysis. We thank Amanpreet Kaur for the help with electron microscopy and Marie Zeuner for careful proof-reading.

\section{Figure Legends}

Figure 1. 3D NFC hydrogel consists of a dense meshwork with heterogeneous pore sizes. A. Calcofluor white staining and fluorescence microscopy was used to visualize the 3D NFC hydrogel under native conditions. A dense and evenly distributed cellulose fibre network was observed. Bar: $300 \mu \mathrm{m}$. B-C. The ultrastructural appearance of the 3D NFC hydrogel was investigated by scanning electron microscopy (SEM) revealing a dense meshwork-like surface structure. Higher magnification image $(C)$ revealed presence of fibers of heterogeneous size. Bars: $300 \mu \mathrm{m}$ and $10 \mu \mathrm{m}$, respectively. D. NFC hydrogels contain nano- and microfibers with a heterogeneous diameter ranging from 0.08 to $9.1 \mu \mathrm{m}$. 
Figure 2. 3D NFC hydrogels support in vitro growth of MSCs. A. AdMSCs were cultivated in $0.2 \%$ NFC hydrogel in standard MSC cultivation medium revealing a typical

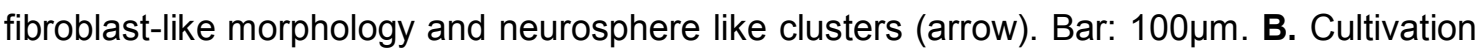
of adMSCs in $0.5 \%$ NFC leads to a homogenous cell population with bipolar morphology (arrowheads). Bar: $50 \mu \mathrm{m}$. C. AdMSCs in $0.5 \%$ NCF hydrogel were fixed using paraformaldehyde and glutaraldehyde and processed for SEM. Data analysis revealed that MSCs interact with the NFC as evidenced by formation of membrane protrusions in direct

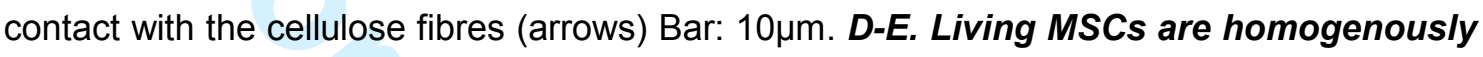
distributed within the NFC matrix. D. AdMSCs were labelled with PKH67 followed by embedding in $0.5 \%$ NFC hydrogel in inserts and fluorescence microscopy revealing high labelling efficiency and individual labelled cells within the hydrogel (arrows). Maximum intensity projection was generated in ImageJ Fiji (LUT: cyan hot). E. High content 3D laser scanning microscopy using the fScan 488 device was applied to visualize the distribution of MSCs in all three dimensions of the NFC hydrogel. Subsequent image analysis in ImageJ Fiji (Volume Viewer Plugin, LUT: cyan hot) revealed isotropic and homogenous cell distribution. Scan area: $10 \mathrm{~mm} \times 10 \mathrm{~mm} \times 1.6 \mathrm{~mm}(\mathrm{x} / \mathrm{y} / \mathrm{z})$, bar: $1 \mathrm{~mm}$.

Figure 3. Different concentrations of 3D NFC hydrogels are biocompatible and support growth of viable human MSCs of different origin. A-B. Confocal and phase contrast imaging of adMSCs within a $0.2 \%$ NFC, stained with Calcein (living cells), Ethidium homodimer-1 (dead cells) and counter-stained with DAPI shows evenly distributed viable cells embedded within the NFC. 3D reconstruction (A) with part of the matrix cut through the y plane reveals supported cells, whilst the maximum projection image (B) shows neither Ethidium homodimer-1 signal nor pyknotic nuclei. C. To assess the influence of 3D cultivation within the NFC hydrogel on cellular viability of human MSCs of different cellular origin, bone marrow MSCs, palatal adipose tissue-derived MSCs and liposuction-derived adipose tissue MSCs were embedded in $0.2 \%$ or $0.5 \%$ 3D NFC hydrogel and assessed for viability (XTT assay). No significant difference in cellular viability was observed in all groups 
cultivated in $0.2 \%$ NFC compared to $2 \mathrm{D}$ controls. A slight but significant reduction of viability was observed in bmMSCs cultivated in the $0.5 \%$ NFC hydrogel. D. MSCs proliferate within the NFC hydrogel. $36 \times 10^{3}$ adMSCs were embedded in $0.2 \%$ or $0.5 \%$ NFC followed by cultivation for $36 \mathrm{~h}$ and XTT. A standard curve with defined cell numbers (10, 1000, 10000, and 100000 cells) was used to normalize the XTT absorption to cell numbers. E. 25000 MSCs were seeded in $0.2 \%$ or $0.5 \%$ NFC followed by retrieval using cellulase. Approximately $100 \%$ were retrieved from $0.2 \%$ NFC, whereas $50 \%$ from $0.5 \%$ NFC. F. Viable MSCs can be retrieved from the 3D NFC hydrogel. AdMSCs were cultivated in $0.2 \%$ NFC hydrogel followed by enzymatic retrieval using cellulase. After replating under standard 2D conditions, an XTT assay was performed to assess the viability of the cells which had previously been cultivated in 3D NFC hydrogel (post 3D) compared to control cells (2D). ** $P<0.01$.

Figure 4. 3D NFC is suitable for osteogenic differentiation of MSCs in $3 D$. A. Liposuction-derived adMSC and adMSCs isolated from palatal adipose tissue were subjected to osteogenic differentiation in $0.2 \%$ NFC for 21 days and subsequently stained for calcium deposition with Alizarin Red S. Images of differentiated MSCs clearly show calcium deposition (magenta), whereas no differentiation was observed in control cells, which appeared red. B. Higher power images showing Alizarin Red S-labelled calcium deposits at cellular level in palatal tissue-derived MSCs. C. NFC control without cells showed only low level of Alizarin staining.

\section{References}

1. R.R. Sharma, K. Pollock, A. Hubel, and D. McKenna: Mesenchymal stem or stromal cells: a review of clinical applications and manufacturing practices. Transfusion 54, 1418 (2014).

2. M. Gnecchi, H. He, N. Noiseux, O.D. Liang, L. Zhang, F. Morello, H. Mu, L.G. Melo, R.E. Pratt, J.S. Ingwall, and V.J. Dzau: Evidence supporting paracrine hypothesis for Akt-modified mesenchymal stem cell-mediated cardiac protection and functional improvement. FASEB J 20, 661 (2006).

3. R.C. Lai, F. Arslan, M.M. Lee, N.S. Sze, A. Choo, T.S. Chen, M. Salto-Tellez, L. Timmers, C.N. Lee, R.M. El Oakley, G. Pasterkamp, D.P. de Kleijn, and S.K. Lim: Exosome secreted by MSC reduces myocardial ischemia/reperfusion injury. Stem Cell Res 4, 214 (2010). 
4. L. Kordelas, V. Rebmann, A.K. Ludwig, S. Radtke, J. Ruesing, T.R. Doeppner, M. Epple, P.A. Horn, D.W. Beelen, and B. Giebel: MSC-derived exosomes: a novel tool to treat therapy-refractory graft-versus-host disease. Leukemia 28, 970 (2014).

5. U. Ben-David, Y. Mayshar, and N. Benvenisty: Large-Scale Analysis Reveals Acquisition of Lineage-Specific Chromosomal Aberrations in Human Adult Stem Cells. Cell Stem Cell 9, 97 (2011).

6. J.J. Bara, R.G. Richards, M. Alini, and M.J. Stoddart: Concise review: Bone marrowderived mesenchymal stem cells change phenotype following in vitro culture: implications for basic research and the clinic. Stem Cells 32, 1713 (2014).

7. V. Turinetto, E. Vitale, and C. Giachino: Senescence in Human Mesenchymal Stem Cells: Functional Changes and Implications in Stem Cell-Based Therapy. Int J Mol Sci 17(2016).

8. S.S. Ho, K.C. Murphy, B.Y. Binder, C.B. Vissers, and J.K. Leach: Increased Survival and Function of Mesenchymal Stem Cell Spheroids Entrapped in Instructive Alginate Hydrogels. Stem Cells Transl Med 5, 773 (2016).

9. A.W. Lund, J.P. Stegemann, and G.E. Plopper: Mesenchymal Stem Cells Sense Three Dimensional Type I Collagen through Discoidin Domain Receptor 1. Open Stem Cell J 1, 40 (2009).

10. O.F. Gardner, G. Musumeci, A.J. Neumann, D. Eglin, C.W. Archer, M. Alini, and M.J. Stoddart: Asymmetrical seeding of MSCs into fibrin-poly(ester-urethane) scaffolds and its effect on mechanically induced chondrogenesis. J Tissue Eng Regen Med (2016).

11. P.M. Favi, R.S. Benson, N.R. Neilsen, R.L. Hammonds, C.C. Bates, C.P. Stephens, and M.S. Dhar: Cell proliferation, viability, and in vitro differentiation of equine mesenchymal stem cells seeded on bacterial cellulose hydrogel scaffolds. Mater Sci Eng C Mater Biol App/ 33, 1935 (2013).

12. A. Cochis, S. Grad, M.J. Stoddart, S. Fare, L. Altomare, B. Azzimonti, M. Alini, and L. Rimondini: Bioreactor mechanically guided 3D mesenchymal stem cell chondrogenesis using a biocompatible novel thermo-reversible methylcellulosebased hydrogel. Sci Rep 7, 45018 (2017).

13. Y. Yamaguchi, J. Ohno, A. Sato, H. Kido, and T. Fukushima: Mesenchymal stem cell spheroids exhibit enhanced in-vitro and in-vivo osteoregenerative potential. BMC Biotechnol 14, 105 (2014).

14. M.A. Serban, Y. Liu, and G.D. Prestwich: Effects of extracellular matrix analogues on primary human fibroblast behavior. Acta Biomater 4, 67 (2008).

15. Y.R. Lou, L. Kanninen, T. Kuisma, J. Niklander, L.A. Noon, D. Burks, A. Urtti, and M. Yliperttula: The use of nanofibrillar cellulose hydrogel as a flexible three-dimensional model to culture human pluripotent stem cells. Stem Cells Dev 23, 380 (2014).

16. M. Bhattacharya, M.M. Malinen, P. Lauren, Y.R. Lou, S.W. Kuisma, L. Kanninen, M. Lille, A. Corlu, C. GuGuen-Guillouzo, O. Ikkala, A. Laukkanen, A. Urtti, and M. Yliperttula: Nanofibrillar cellulose hydrogel promotes three-dimensional liver cell culture. J Control Release 164, 291 (2012).

17. M.M. Malinen, L.K. Kanninen, A. Corlu, H.M. Isoniemi, Y.R. Lou, M.L. Yliperttula, and A.O. Urtti: Differentiation of liver progenitor cell line to functional organotypic cultures in 3D nanofibrillar cellulose and hyaluronan-gelatin hydrogels. Biomaterials 35, 5110 (2014).

18. M. Dominici, K. Le Blanc, I. Mueller, I. Slaper-Cortenbach, F. Marini, D. Krause, R. Deans, A. Keating, D. Prockop, and E. Horwitz: Minimal criteria for defining multipotent mesenchymal stromal cells. The International Society for Cellular Therapy position statement. Cytotherapy 8, 315 (2006).

19. J. Schindelin, I. Arganda-Carreras, E. Frise, V. Kaynig, M. Longair, T. Pietzsch, S. Preibisch, C. Rueden, S. Saalfeld, B. Schmid, J.Y. Tinevez, D.J. White, V. Hartenstein, K. Eliceiri, P. Tomancak, and A. Cardona: Fiji: an open-source platform for biological-image analysis. Nat Methods 9, 676 (2012). 
20. A. Kaus, D. Widera, S. Kassmer, J. Peter, K. Zaenker, C. Kaltschmidt, and B. Kaltschmidt: Neural stem cells adopt tumorigenic properties by constitutively activated NF-kappaB and subsequent VEGF up-regulation. Stem Cells Dev 19, 999 (2010).

21. O.W. Petersen, L. Rønnov-Jessen, A.R. Howlett, and M.J. Bissell: Interaction with basement membrane serves to rapidly distinguish growth and differentiation pattern of normal and malignant human breast epithelial cells. Proceedings of the National Academy of Sciences of the United States of America 89, 9064 (1992).

22. C.M. Yang, Y.J. Huang, and S.H. Hsu: Enhanced Autophagy of Adipose-Derived Stem Cells Grown on Chitosan Substrates. Biores Open Access 4, 89 (2015).

23. H.K. Kleinman and G.R. Martin: Matrigel: Basement membrane matrix with biological activity. Seminars in Cancer Biology 15, 378 (2005).

24. J.R. Paletta, F. Mack, H. Schenderlein, C. Theisen, J. Schmitt, J.H. Wendorff, S. Agarwal, S. Fuchs-Winkelmann, and M.D. Schofer: Incorporation of osteoblasts (MG63) into 3D nanofibre matrices by simultaneous electrospinning and spraying in bone tissue engineering. Eur Cell Mater 21, 384 (2011).

25. M. Bhattacharya, M.M. Malinen, P. Lauren, Y.-R. Lou, S.W. Kuisma, L. Kanninen, M. Lille, A. Corlu, C. GuGuen-Guillouzo, O. Ikkala, A. Laukkanen, A. Urtti, and M. Yliperttula: Nanofibrillar cellulose hydrogel promotes three-dimensional liver cell culture. Journal of Controlled Release 164, 291 (2012).

26. H. Paukkonen, A. Ukkonen, G. Szilvay, M. Yliperttula, and T. Laaksonen: Hydrophobin-nanofibrillated cellulose stabilized emulsions for encapsulation and release of BCS class II drugs. European Journal of Pharmaceutical Sciences 100, 238 (2017).

27. D.J. Modulevsky, C.M. Cuerrier, and A.E. Pelling: Biocompatibility of Subcutaneously Implanted Plant-Derived Cellulose Biomaterials. PLoS One 11, e0157894 (2016).

28. V.R. Lopes, C. Sanchez-Martinez, M. Stromme, and N. Ferraz: In vitro biological responses to nanofibrillated cellulose by human dermal, lung and immune cells: surface chemistry aspect. Part Fibre Toxicol 14, 1 (2017). 


\section{Figure 1}

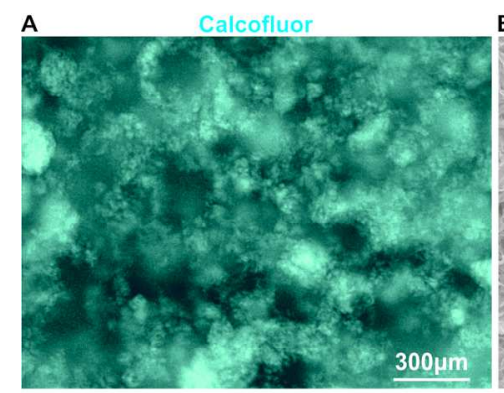

B

C
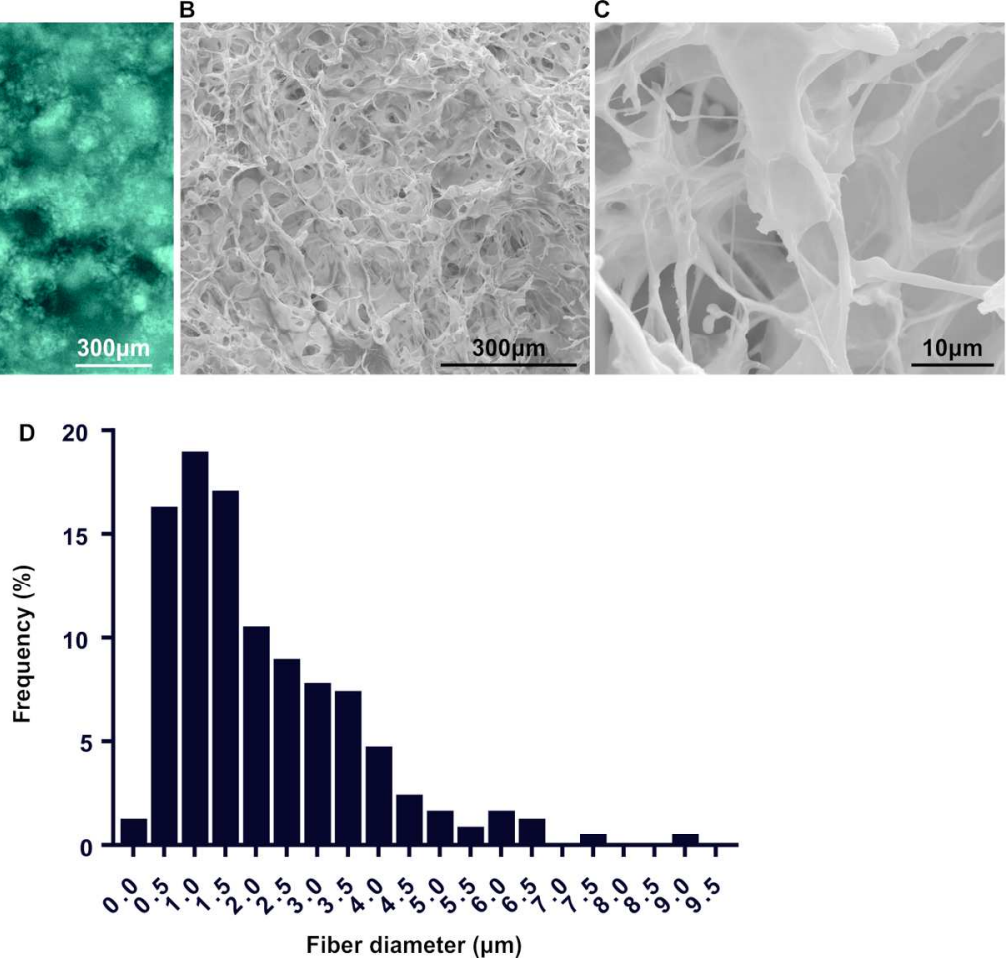

Figure 1. 3D NFC hydrogel consists of a dense meshwork with heterogeneous pore sizes. A. Calcofluor white staining and fluorescence microscopy was used to visualize the 3D NFC hydrogel under native conditions. A dense and evenly distributed cellulose fibre network was observed. Bar: $300 \mu \mathrm{m}$. B-C. The ultrastructural appearance of the 3D NFC hydrogel was investigated by scanning electron microscopy (SEM) revealing a dense meshwork-like surface structure. Higher magnification image $(C)$ revealed presence of fibers of heterogeneous size. Bars: $300 \mu \mathrm{m}$ and $10 \mu \mathrm{m}$, respectively. D. NFC hydrogels contain nano- and microfibers with a heterogeneous diameter ranging from 0.08 to $9.1 \mu \mathrm{m}$.

$$
159 \times 121 \mathrm{~mm}(300 \times 300 \mathrm{DPI})
$$




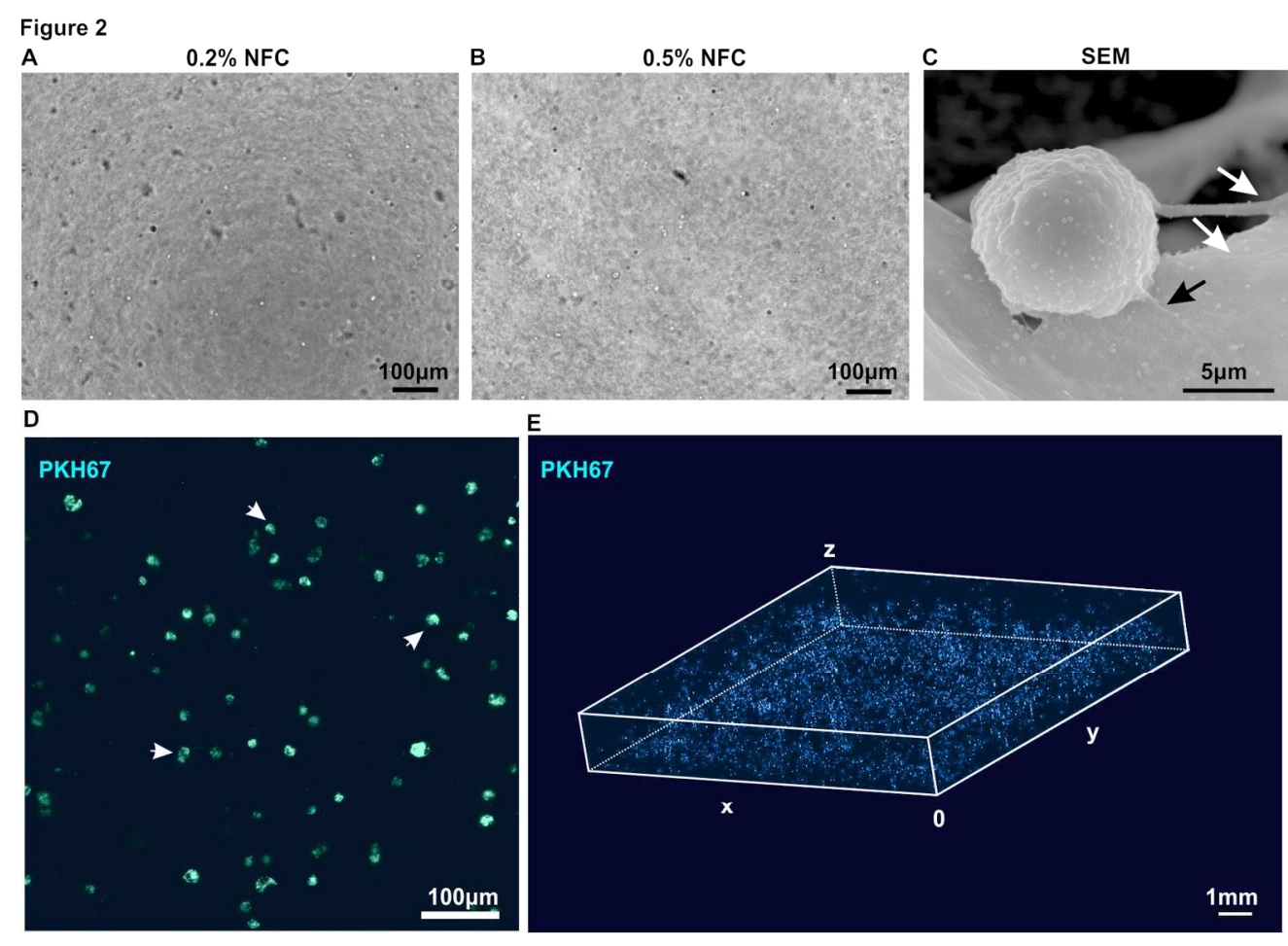

Figure 2. 3D NFC hydrogels support in vitro growth of MSCs. A. AdMSCs were cultivated in $0.2 \%$ NFC hydrogel in standard MSC cultivation medium revealing a typical fibroblast-like morphology and neurosphere like clusters (arrow). Bar: $100 \mu \mathrm{m}$. B. Cultivation of adMSCs in $0.5 \%$ NFC leads to a homogenous cell population with bipolar morphology (arrowheads). Bar: $50 \mu \mathrm{m}$. C. AdMSCs in $0.5 \%$ NCF hydrogel were fixed using paraformaldehyde and glutaraldehyde and processed for SEM. Data analysis revealed that MSCS interact with the NFC as evidenced by formation of membrane protrusions in direct contact with the cellulose fibres (arrows) Bar: 10 $\mu \mathrm{m}$. D-E. Living MSCs are homogenously distributed within the NFC matrix. D. AdMSCs were labelled with PKH67 followed by embedding in $0.5 \%$ NFC hydrogel in inserts and fluorescence microscopy revealing high labelling efficiency and individual labelled cells within the hydrogel (arrows).

Maximum intensity projection was generated in ImageJ Fiji (LUT: cyan hot). E. High content 3D laser scanning microscopy using the fScan 488 device was applied to visualize the distribution of MSCs in all three dimensions of the NFC hydrogel. Subsequent image analysis in ImageJ Fiji (Volume Viewer Plugin, LUT: cyan hot) revealed isotropic and homogenous cell distribution. Scan area: $10 \mathrm{~mm} \times 10 \mathrm{~mm} \times 1.6 \mathrm{~mm}(\mathrm{x} / \mathrm{y} / \mathrm{z})$, bar: $1 \mathrm{~mm}$. 
Figure 3
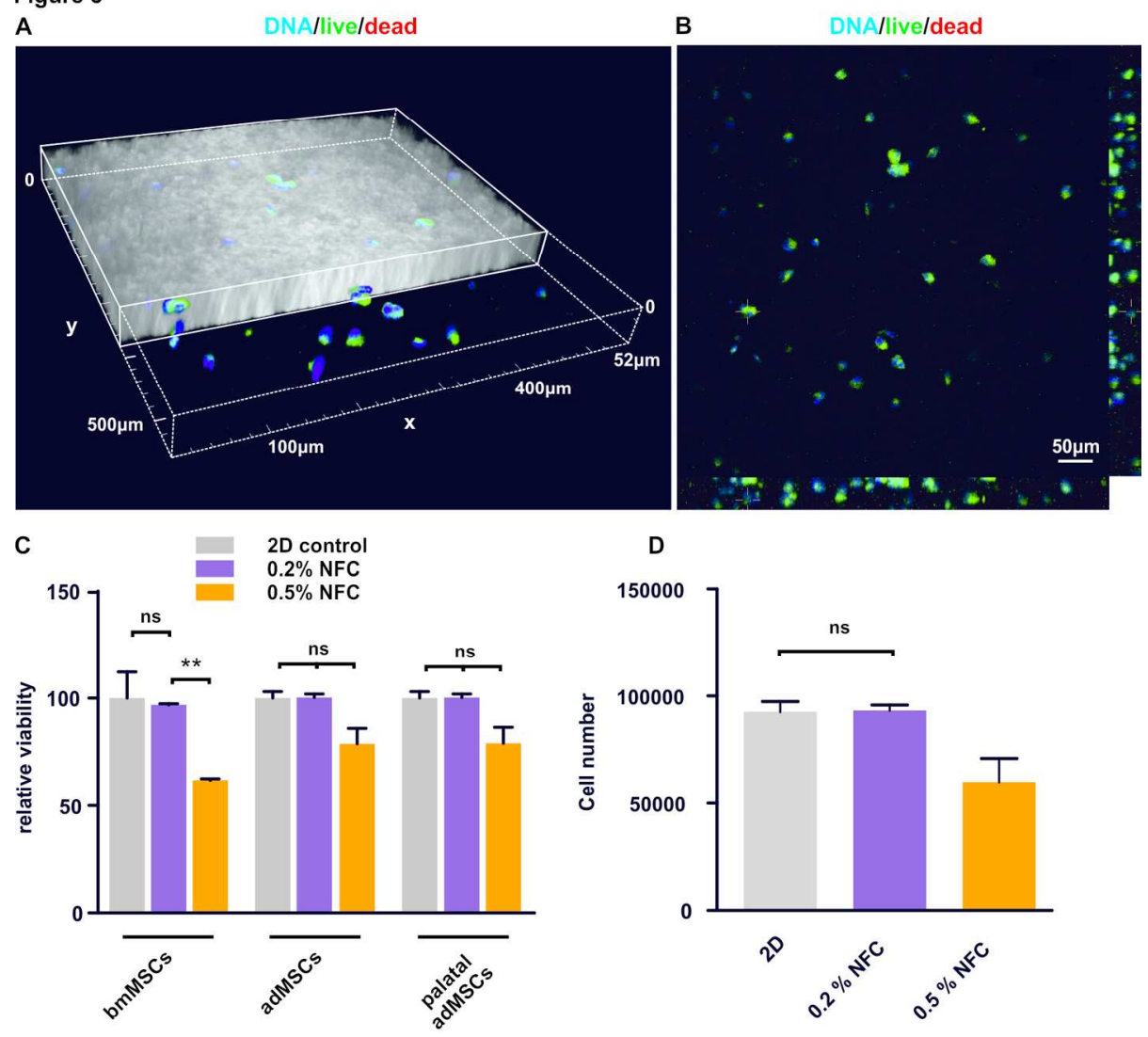

D

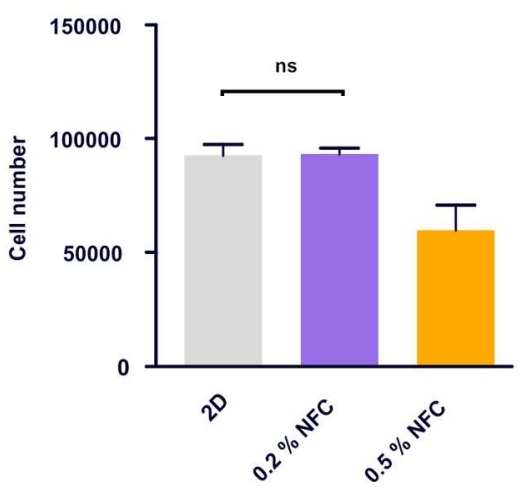

E

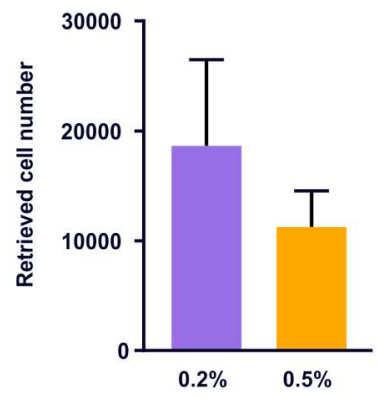

$\mathbf{F}$

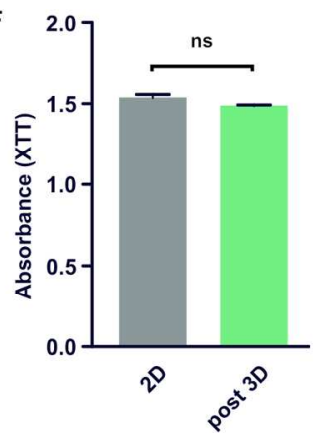

Figure 3. Different concentrations of 3D NFC hydrogels are biocompatible and support growth of viable human MSCs of different origin. A-B. Confocal and phase contrast imaging of adMSCs within a $0.2 \%$ NFC, stained with Calcein (living cells), Ethidium homodimer-1 (dead cells) and counter-stained with DAPI shows evenly distributed viable cells embedded within the NFC. 3D reconstruction (A) with part of the matrix cut

through the y plane reveals supported cells, whilst the maximum projection image (B) shows neither Ethidium homodimer-1 signal nor pyknotic nuclei. C. To assess the influence of 3D cultivation within the NFC hydrogel on cellular viability of human MSCs of different cellular origin, bone marrow MSCs, palatal adipose tissue-derived MSCs and liposuction-derived adipose tissue MSCs were embedded in $0.2 \%$ or $0.5 \%$ 3D NFC hydrogel and assessed for viability (XTT assay). No significant difference in cellular viability was observed in all groups cultivated in $0.2 \%$ NFC compared to 2D controls. A slight but significant reduction of viability was observed in bmMSCs cultivated in the $0.5 \%$ NFC hydrogel. D. MSCs proliferate within the NFC hydrogel. 36000 adMSCs were embedded in $0.2 \%$ or $0.5 \%$ NFC followed by cultivation for $36 \mathrm{~h}$ and XTT. A standard curve with defined cell numbers (11, 1000, 10000, and 10000 cells) was used to normalize the XTT 
absorption to cell numbers. E. 25000 MSCs were seeded in $0.2 \%$ or $0.5 \%$ NFC followed by retrieval using cellulase. Approximately $100 \%$ were retrieved from $0.2 \%$ NFC, whereas $50 \%$ from $0.5 \%$ NFC. F. Viable MSCs can be retrieved from the 3D NFC hydrogel. AdMSCs were cultivated in $0.2 \%$ NFC hydrogel followed by enzymatic retrieval using cellulase. After replating under standard 2D conditions, an XTT assay was performed to assess the viability of the cells which had previously been cultivated in 3D NFC hydrogel (post 3D) compared to control cells (2D). ** $\mathrm{P}<0.01$.

$156 \times 204 \mathrm{~mm}(300 \times 300$ DPI $)$ 


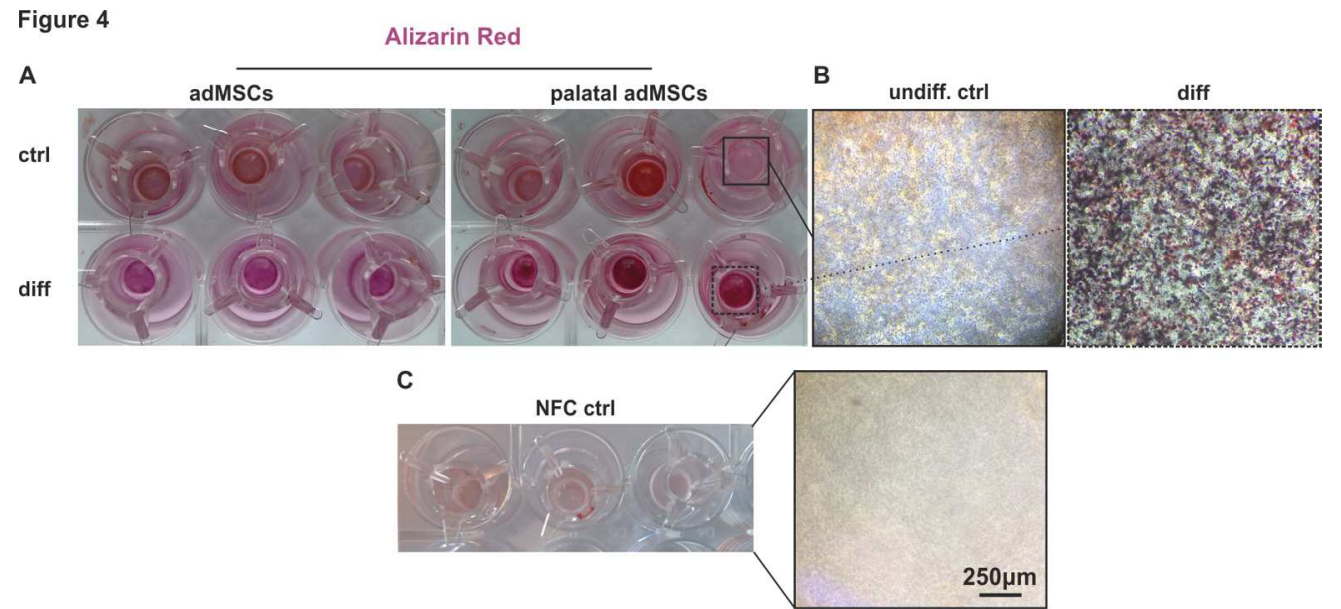

Figure 4. 3D NFC is suitable for osteogenic differentiation of MSCs in 3D. A. Liposuction-derived adMSC and adMSCs isolated from palatal adipose tissue were subjected to osteogenic differentiation in $0.2 \%$ NFC for 21 days and subsequently stained for calcium deposition with Alizarin Red S. Images of differentiated MSCs clearly show calcium deposition (magenta), whereas no differentiation was observed in control cells, which appeared red. B. Higher power images showing Alizarin Red S-labelled calcium deposits at cellular level in palatal tissue-derived MSCs. C. NFC control without cells showed only low level of Alizarin staining.

$156 \times 72 \mathrm{~mm}(300 \times 300 \mathrm{DPI})$ 